\title{
Working memory and spatial judgments: Cognitive load increases the central tendency bias
}

\author{
Sarah R. Allred ${ }^{1} \cdot$ L. Elizabeth Crawford ${ }^{2} \cdot$ Sean Duffy $^{1} \cdot$ John Smith $^{3}$
}

Published online: 15 April 2016

(C) Psychonomic Society, Inc. 2016

\begin{abstract}
Previous work demonstrates that memory for simple stimuli can be biased by information about the distribution of which the stimulus is a member. Specifically, people underestimate values greater than the distribution's average and overestimate values smaller than the average. This is referred to as the central tendency bias. This bias has been explained as an optimal use of both noisy sensory information and category information. In largely separate literature, cognitive load (CL) experiments attempt to manipulate the available working memory of participants in order to observe the effect on choice or judgments. In two experiments, we demonstrate that participants under high cognitive load exhibit a stronger central tendency bias than when under a low cognitive load. Although not anticipated at the outset, we also find that judgments exhibit an anchoring bias not described previously.
\end{abstract}

We thank Roberto Barbera and Noha Emara for helpful comments. J.S. thanks Biblioteca de Catalunya. This project was supported by Rutgers University Research Council Grants (\#202167, \#202110, \#202256, \#202204) and NSF CAREER BCS 0954749.

Electronic supplementary material The online version of this article (doi:10.3758/s13423-016-1039-0) contains supplementary material, which is available to authorized users.

Sean Duffy

seduffy@scarletmail.rutgers.edu

1 Department of Psychology, Rutgers University-Camden, 343 Armitage Hall, 511 N. 5th Street, Camden, NJ 08102, USA

2 Department of Psychology, The University of Richmond, Richmond, VA, USA

3 Department of Economics, Rutgers University-Camden, Camden, NJ, USA
Keywords Judgment $\cdot$ Memory $\cdot$ Anchoring $\cdot$ Working memory $\cdot$ Cognitive constraints $\cdot$ Cognitive load

Memory is an essential function, yet a large body of research suggests that memory exhibits systematic biases. One wellknown bias is central tendency, when individuals remember stimuli as being more typical of the category of which they are members (Goldstone, 1994). Once considered a perceptual or mnemonic distortion (e.g., Poulton, 1989), this bias has been described as resulting from an adaptive, Bayesian process that combines inexact memories of individual stimuli with prior knowledge about the distribution of the category. Combining information in this manner improves the average accuracy of judgments, even though it introduces bias into individual estimates.

Huttenlocher and colleagues (Crawford, Huttenlocher, \& Engebretson, 2000; Crawford, Huttenlocher, \& Hedges, 2006; Duffy, Huttenlocher, \& Crawford, 2006; Duffy, Huttenlocher, Hedges, \& Crawford, 2010; Huttenlocher, Hedges, \& Vevea, 2000) proposed the category adjustment model (CAM). In it, categories are summarized as distributions of values along some stimulus dimension, such as size or shape, and a stimulus as a particular value along this dimension. For most categories, the average value of the distribution is the prototypical value (Duffy \& Crawford, 2008).

The CAM is similar to other Bayesian models that have been used to explain biases in memory for size estimation (Ashourian \& Loewenstein, 2011), time perception (Jazayeri \& Shadlen, 2010), and hue bias (Olkkonen \& Allred, 2014; Olkkonen, McCarthy, \& Allred, 2014). Research has found these effects extend to judgments of realistic and familiar objects (Hemmer \& Steyvers, 2009a, 2009b). Despite the success of CAM, there are mixed results regarding whether participants successfully execute the optimal Bayesian judgments 
by employing all available and relevant information (Sailor \& Antoine, 2005; Xu \& Griffiths, 2010).

In this paper we study the central tendency bias by employing a cognitive load (CL) manipulation. Studies employing CL manipulations often direct participants to engage in memorization tasks in parallel with making a decision within another domain. The memorization tasks manipulate available working memory. Extensive literature documents that cognitive resources are bounded and that increasing CL can affect judgments (Cornelissen, Dewitte, \& Warlop, 2011; Hinson, Jameson, \& Whitney, 2003; Swann, Hixon, SteinSeroussi, \& Gilbert, 1990; Van den Bos, Peters, Bobocel, \& Ybema, 2006), limit the ability to process information (Gilbert, Pelham, \& Krull, 1988), decrease self-control (Mann \& Ward, 2007; Shiv \& Fedorikhin, 1999; Ward \& Mann, 2000), affect strategic behavior (Allred, Duffy, \& Smith, 2016; Duffy \& Smith, 2014; Duffy, Owens \& Smith, unpublished), increase the assimilation effect (Martin, Seta, \& Crelia, 1990), affect duration judgments (Block, Hancock, \& Zakay, 2010), and prompt stereotyping (Wigboldus, Sherman, Franzese, \& van Knippenberg, 2004).

We are not the first to examine the effect of CL on visual judgments. Allen, Baddeley, and Hitch (2006) found that memorization of numbers increased $\mathrm{CL}$ and reduced the accuracy of color and shape recall. Morey and Cowan (2004) found reduced performance on a visual memory task under a CL that required the memorization of seven random digits. Morey and Bieler (2013) found that participants who are required to recall different tones have a reduced accuracy of recall of colors and shapes. Zokaei, Heider, and Husain (2014) found that CL affects performance in a variety of visual judgment tasks. Surprisingly, Cocchi et al. (2011) found that participants under a high visual working memory CL exhibited better performance on a visual memory task than participants under a low visual working memory CL. However, to our knowledge, ours is the first paper to examine the effect of $\mathrm{CL}$ on the central tendency bias.

In our two experiments we examine the effect of CL on the degree to which category information influences stimulus estimates. We employ a sequential line estimation task in which participants observe and reproduce a series of lines that vary in length under conditions of high or low CL. Bias may increase if $\mathrm{CL}$ degrades the precision of the memories of individual stimuli; or, CL might interfere with the underlying ability to combine information about the stimuli and category, resulting in decreased bias. However, it is possible that CL may have no effect on bias if $\mathrm{CL}$ is unrelated to the underlying cognitive processes that produce central tendency in judgment.

But, also recognizing a possible limitation in prior research using this paradigm, we were concerned that our data could exhibit anchoring effects (see Furnham \& Boo, 2011, for a review of the anchoring literature). Specifically, when reproducing lines, it is possible that the starting length of a reproduction line might affect resulting estimates. Literature has found that participants produce biased judgments in a wide variety of settings due to the influence of an uninformative, constant anchor. In their Experiment 2c, Epley and Gilovich (2006) examined the effects of CL on anchoring in a numerical response setting. The authors found that participants under high CL were more sensitive to the anchor than were participants under low CL. LeBoeuf and Shafir (2006) examined whether anchoring effects can be found in the judgment of physical quantities. The authors found anchoring effects in judgments of length, weight, and loudness. Prior studies on the central tendency bias used a constant starting length (Crawford, Huttenlocher, \& Engebretson, 2000; Huttenlocher et al., 2000) or a randomized starting length but did not analyze its effects (Duffy \& Crawford, 2008). Therefore, we explore anchoring effects in judgments of length, which has not yet been studied in a setting designed to study the central tendency bias.

\section{Experiment 1: randomly ordered CL}

\section{Method}

\section{Participants}

Thirty-three undergraduates (20 females, 13 males) participated. In this and Experiment 2, participants were tested in a windowless laboratory and received partial course credit.

\section{Design and procedure}

Each trial started with participants viewing a two-digit (low cognitive load) or six-digit (high cognitive load) number that they were instructed to commit to memory. Each of the two 0 or six digits were randomly selected from the integers 0 to 9 , although the initial digit was never 0 . The number was presented for 5 seconds. The participants then observed a line for 1.5 seconds. We refer to this line as the target line. Target lines ranged from 96 to 352 pixels at 9 unique stimulus sizes that varied in 32 pixel increments. After a 1.5 -second delay with a blank screen, participants saw a second, adjustable line. We refer to the initial length of the adjustable line as the start line length. Unlike most prior studies (which used the same start line length on all trials), the length of the start line was randomly drawn from a uniform distribution ranging from the length of the shortest target line (96 pixels) to the longest target line (352 pixels). The participants increased or decreased the length of this adjustable line by pressing the mouse buttons in order to match their memory of the length of the target line. After participants were satisfied with the length of their adjustable line, they pressed Enter. We refer to this as the response line. 
Subsequently, participants were prompted to type the twoor six-digit number. After pressing Enter they repeated this process with the next trial. The order of trials was randomly presented both by line length and CL. There were a total of 90 trials, with 10 lines from each of the nine possible stimulus values. Of the 10 trials at each stimulus value, five were conducted under high $\mathrm{CL}$ and five were conducted under low $\mathrm{CL}$.

\section{Results and discussion}

We first performed manipulation checks to examine whether the difficulty of the memorization task affected the ability to remember the number. As expected, participants under high $C L$ were less accurate $(79.19 \%)$ on the number memorization task than they were on low CL trials $(91.25 \%), t(32)=5.78, p$ $<.001$, suggesting that participants found the number in highload trials more difficult to remember than those in low-load trials. We also examined whether any individuals performed extremely poorly on the memorization task. Such poor performance could indicate that the participant did not devote sufficient cognitive resources to remember the numbers, and we would exclude these participants from the analysis. All participants satisfied our inclusion criterion of performing more than $50 \%$ correct on the high CL memorization trials.

In order to examine overall accuracy, we calculated absolute bias as the absolute difference between the target and response line length. Participants were less accurate under high CL $(M=29.76, S D=30.11)$ than low CL $(M=25.18$, $S D=25.06), t(2968)=-4.50, p<.001$. Despite this, participants did not adjust their responses, as measured by absolute difference between the start and the response, differently while under high CL $(M=92.91, S D=68.57)$ than while under low $\mathrm{CL}(M=93.95, S D=72.08), t(2968)=0.40, p=.69$.

We also calculated the response bias as the difference between the target and response line length. Figure 1 shows average response bias on the vertical axis and the length of the target line on the horizontal axis for the two within-subject conditions (high and low CL). For each participant, we ran a regression of bias by target line length to obtain slopes for each participant separately for the high- and low-load conditions. From this, we obtained slopes for each participant under both load conditions. We tested the hypothesis that slopes were steeper under high rather than low load using a pairedsamples $t$ test. We found that average individual slopes were steeper under high CL $(M=-0.16, S D=0.02)$ than low CL ( $M$ $=-0.05, S D=0.02), t(32)=6.45, p<.001$.

We further analyzed the central tendency effects, possible anchoring effects, and the CL effects by performing a repeatedmeasures regression with response bias as the dependent variable for every trial rather than data aggregated by participant and stimulus size as in the prior analysis. We analyzed these effects in the same specifications because it was not obvious

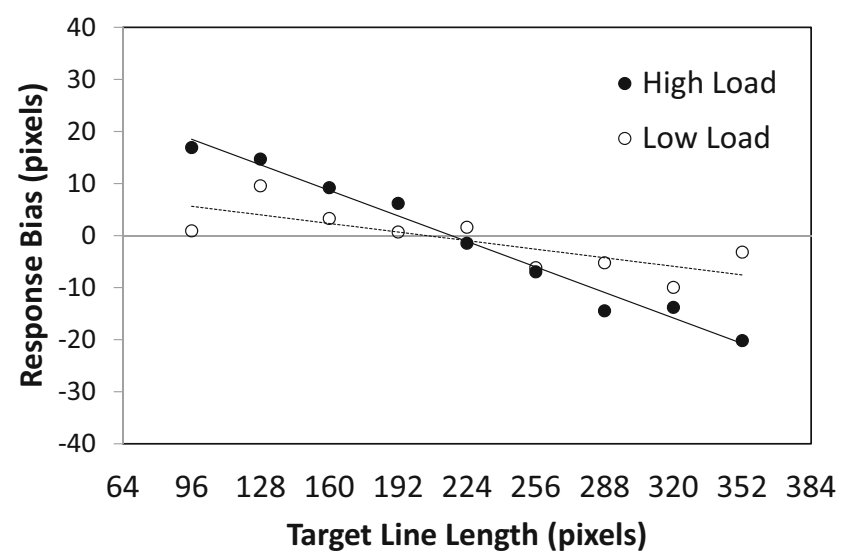

Fig. 1 Cognitive load affects the magnitude of the central tendency bias in Experiment 1. In this and the following figures, response bias (target line length minus the response line length) is plotted as a function of the target line length under low cognitive load (open symbols) and under high cognitive load (solid symbols). Data points are averages across all trials for all participants. The solid (high load) and dashed (low load) lines are best fitting lines. The solid horizontal line represents a response bias of zero, or veridical memory

that the anchoring effects were robust to the specifications that also accounted for the central tendency bias and the CL.

We included independent variables that characterized the $\mathrm{CL}$ associated with the observation, the target line length, and the length of the start line. In particular, we included a categorical variable, high load, which indicated whether the decision was taken under high or low CL. We also included a centered target variable, which was defined to be the length of the target line minus the mean of the lines. Finally, in order to investigate possible anchoring effects, we included the start variable, which was the length of the start line.

We included specifications that accounted for the repeated nature of our data. In these specifications, we estimated a compound symmetry covariance matrix clustered by participant. This implied that any two observations involving a participant were assumed to be correlated, but any observations involving different participants were assumed to be independent. We reported the unstandardized coefficient estimates and standard errors. Reporting the unstandardized coefficient estimates, rather than the standardized estimates, seemed to be appropriate since the variables of interest - centered target and start-were both measured in pixels.

Finally, as our data possibly exhibited a nonlinear relationship, we offered a specification that excluded the longest and shortest target line values. ${ }^{1}$ We summarized this analysis in Table 1 .

The high-load variable estimates were not significantly different from zero. This suggested that CL does not lead to an overestimation of all lines or an underestimation of all lines.

\footnotetext{
${ }^{1}$ A reviewer noted the inverted $S$ shape of the low CL trials. In online supplementary materials, we present a detailed analysis of this curvilinear effect with cubic terms. This analysis leads to the same qualitative conclusions.
} 
Table 1 Dependent variable: response bias (Experiment 1)

(1) (2)

\begin{tabular}{|c|c|c|c|c|c|c|}
\hline \multirow[b]{2}{*}{ Variable } & \multicolumn{2}{|l|}{ (1) } & \multicolumn{2}{|l|}{ (2) } & \multicolumn{2}{|l|}{ (3) } \\
\hline & $B$ & $S E$ & $B$ & $S E$ & $B$ & $S E$ \\
\hline High load & -4.024 & 3.815 & -4.539 & 3.658 & -2.408 & 3.920 \\
\hline Centered target & $-0.072 * * *$ & 0.0115 & $-0.072 * * *$ & 0.011 & $-0.123 * * *$ & 0.015 \\
\hline High load $*$ Centered target & $-0.117 * * *$ & 0.0163 & $-0.117 * * *$ & 0.0156 & $-0.0613 * *$ & 0.0214 \\
\hline Start & $0.0605 * * *$ & 0.0108 & $0.057 * * *$ & 0.0104 & $0.064 * * *$ & 0.0112 \\
\hline High load $*$ Start & 0.0180 & 0.0151 & 0.0201 & 0.0145 & 0.0115 & 0.0156 \\
\hline Intercept & $-15.30 * * *$ & 2.732 & $-14.56^{* * *}$ & 3.261 & $-16.02 * * *$ & 3.527 \\
\hline AIC & $29,852.8$ & $29,650.4$ & $22,795.3$ & & & \\
\hline Repeated measures & No & Yes & Yes & & & \\
\hline Observations & 2,970 & 2,970 & 2,310 & & & \\
\hline
\end{tabular}

The repeated measures regressions assume a compound symmetry covariance matrix. Specification (1) does not include the repeated measures, specification (2) includes the repeated measures, and specification (3) includes the repeated measures but excludes the observations involving the longest and shortest target lines. $N=2,970: 90$ judgments from 33 participants. $N=2,310: 70$ judgments from 33 participants. AIC refers to the Akaike information criterion (Akaike, 1974)

$B$ is the unstandardized regression coeficient, $S E$ is the standard error of the coefficient estimate

$* p<.05 * * p<.01 * * * p<.001$

The negative centered-target estimates suggested that participants overestimated smaller lines and underestimated longer lines. In other words, we found that judgments were consistent with the central tendency bias. The negative high-load-centered-target interaction estimates suggested that the central tendency bias was stronger for participants under high CL. We therefore found evidence that the CL affects the extent of the central tendency bias.

Across each specification, the positive coefficient estimate of the start variable suggested evidence of anchoring effects. However, we did not find evidence that these anchoring effects were affected by the CL. To address the statistically unlikely possibility that the observed anchoring effects resulted from a biased start length, we investigated the correlation between the target line length and the start line length. We did not find evidence that the start line length was correlated with the target line length, $r(2,968)=-0.0049, p=.79$.

One potential criticism ${ }^{2}$ of Experiment 1 is that the CL manipulation was unusually effective because the order of the load trials was randomized. In other words, it is possible that the high load trials surprised or increased the anxiety experienced by the participants, and this lead to differences between the high- and low-load trials. Therefore, we ran a second experiment where, rather than presenting the CL trials in a random order, we presented the participants with a CL that alternated between high and low across the experiment. This patterned presentation might help participants know what type of CL to anticipate and to prepare accordingly. However, the

\footnotetext{
${ }^{2}$ In online supplementary materials, we describe another experiment (1A) where we directed the subjects to memorize letters rather than numbers. These results are qualitatively similar to those in Experiment 1.
}

lengths of target lines were still randomly selected from the distribution of the nine target line lengths.

\section{Experiment 2: alternating $\mathbf{C L}$}

\section{Method}

\section{Participants}

Forty undergraduates (25 females, 15 males) participated.

\section{Design and procedure}

The procedure was identical to Experiment 1, except that the CL treatment alternated between the 2 and 6 digit numbers. Every participant was first presented with a 2 digit number.

\section{Results and discussion}

Data were analyzed as in Experiment 1. All participants met our inclusion criteria. Under high CL, participants were less accurate in the number memorization task $(75.56 \%)$ than they were under low load $(90.11 \%), t(39)=6.47, p<.001$. Under high CL, responses were less accurate $(M=32.70, S D=$ $39.85)$ than under low CL $(M=28.06, S D=26.13), t(3598)$ $=4.13, p<.001$. Participants under high CL adjusted less $(M=$ 91.90, $S D=75.03)$ than when under low $\mathrm{CL}(M=96.81, S D=$ $70.52), t(3598)=2.02, p=.04$.

Figure 2 illustrates response bias by cognitive load and target line length. To determine whether the slope under high load was steeper than under low load, we performed an analysis identical 


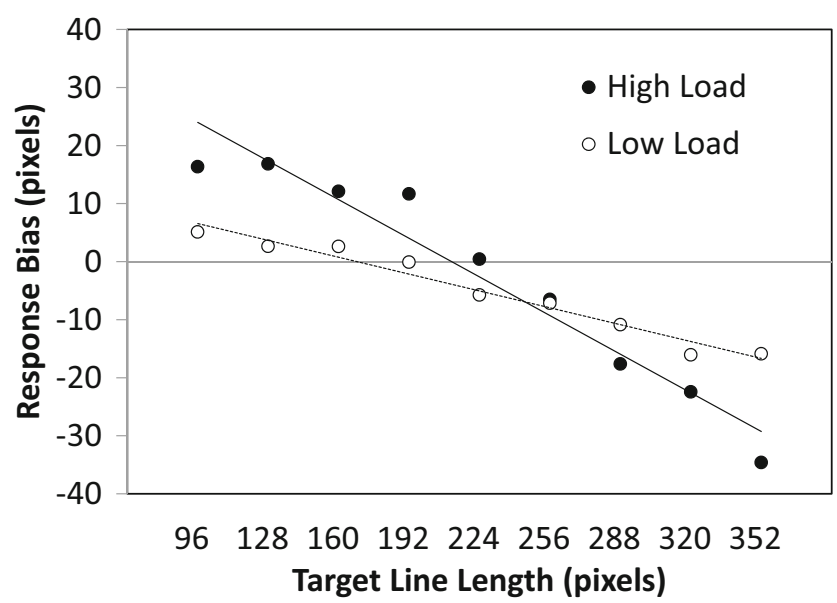

Fig. 2 Cognitive load affects the magnitude of the central tendency bias in the alternating load task (Experiment 2)

to that in Experiment 1. We found the slope was steeper in the high CL condition $(M=-0.20, S D=0.03)$ than in the low $\mathrm{CL}$ condition $(M=-0.09, S D=0.02), t(39)=4.71, p<.001$.

Additionally, we performed a regression analysis identical to that performed for Experiment 1. This analysis is summarized in Table 2. Again, despite the different participants and design, we observed the same qualitative results as in Experiment 1. We found no relationship between the highload variable and response bias. We found evidence of the central tendency bias and that it is more extreme under high CL. Finally, we found evidence of anchoring effects but found no evidence that $\mathrm{CL}$ affected it. To investigate the possibility that a biased start line led to these anchoring effects, we examined the correlation between the target line and the start line. We did not find a significant correlation between the start line and the target line, $r(3,598)=-.03, p=0.07$.

\section{General discussion}

In two experiments, we demonstrated that cognitive load (CL) affects simple judgments of length. Specifically, we found that participants under high CL exhibited stronger central tendency biases than they did when under low CL. Although previous studies showed CL affects visual judgments, to our knowledge we are the first to demonstrate that $\mathrm{CL}$ affects the central tendency bias. We also established that in addition to the central tendency bias there were anchoring effects related to the starting length of the reproduction line, although this bias did not systematically interact with CL.

These results regarding CL and central tendency are of broad interest to those studying memory. Experiments in many stimulus dimensions examine the effect of stimulus distributions on memory (Huttenlocher et al., 2000; Olkkonen et al., 2014). Both the CAM and other Bayesian models demonstrate that biases in memory are well-characterized by combining noisy sensory information with prior information about the distribution of stimuli. Here we add to this literature by suggesting that cognitive load - a variable unrelated to any particular stimulus distribution - magnifies the central tendency bias. Prior research on the central tendency bias never accounted for the possible effect of varying available cognitive resources on estimating categorized stimuli; and, yet, there are many situations and contexts in which people must estimate stimuli while their cognitive resources are limited, for

Table 2 Dependent variable: response bias (Experiment 2)

\begin{tabular}{|c|c|c|c|c|c|c|}
\hline \multirow[b]{2}{*}{ Variable } & \multicolumn{2}{|l|}{ (1) } & \multicolumn{2}{|l|}{ (2) } & \multicolumn{2}{|l|}{ (3) } \\
\hline & $B$ & $S E$ & $B$ & $S E$ & $B$ & $S E$ \\
\hline High load & 2.349 & 4.073 & 2.213 & 3.884 & 3.5211 & 4.2875 \\
\hline Centered target & $-0.0895 * * *$ & 0.0122 & $-0.0933 * * *$ & 0.0116 & $-0.105^{* * *}$ & 0.0171 \\
\hline High load $*$ Centered target & $-0.1097 * * *$ & 0.01738 & $-0.102 * * *$ & 0.0166 & $-0.104 * * *$ & 0.0236 \\
\hline Start & $0.0699 * * *$ & 0.0114 & $0.0693 * * *$ & 0.0109 & $0.0654 * * *$ & 0.01205 \\
\hline High load $*$ Start & 0.000071 & 0.0162 & 0.000656 & 0.0155 & 0.00342 & 0.0170 \\
\hline Intercept & $-21.175 * * *$ & 2.8712 & $-21.015 * * *$ & 3.487 & $-20.179 * * *$ & 3.8679 \\
\hline AIC & $37,315.5$ & $37,039.1$ & $28,673.7$ & & & \\
\hline Repeated measures & No & Yes & Yes & & & \\
\hline Observations & 3,600 & 3,600 & 2,800 & & & \\
\hline
\end{tabular}

The repeated measures regressions assume a compound symmetry covariance matrix. Specification (1) does not include the repeated measures, specification (2) includes the repeated measures, and specification (3) includes the repeated measures but excludes the observations involving the longest and shortest target lines. $N=3,600: 90$ judgments from 40 participants. $N=2,800: 70$ judgments from 40 participants. AIC refers to the Akaike information criterion (Akaike, 1974)

$B$ is the unstandardized regression coeficient, $S E$ is the standard error of the coefficient estimate

$* p<.05 * * p<.01 * * * p<.001$ 
example, under dual-task conditions or while attention is otherwise distracted. Factoring CL into models of estimation may help improve fit and explain behavior.

Although we have shown that CL increases the central tendency bias, the precise mechanism through which this occurs is not yet understood. There are several possible explanations. The most likely mechanism by which $\mathrm{CL}$ increases bias is through limiting cognitive resources in working memory for maintaining a precise representation of the target line length. Given a coarse memory trace under high CL, it would be rational to use information about the distribution as a whole to compensate for the decrease in memory precision. This explanation is consistent with the CAM and other Bayesian models that explain the central tendency bias. However, a second possibility is that memories for individual stimuli and the average of the distribution could be more difficult to separate under high CL, leading to an estimate that blends the memory of the average of the distribution (or some subset of prior stimuli) with the target line length, which may be stronger under high CL. It is also possible that both of these processes work synergistically to produce the observed effect. Although the precise mechanism explaining increased bias under high $\mathrm{CL}$ is a direction for future research, it is clear that the availability of cognitive resources in working memory affects participants' ability to accurately reproduce stimuli.

We also found anchoring effects in judgments of length. To our knowledge, these findings are the first to find anchoring effects in investigations of the central tendency bias. Here we find significant anchoring effects, in that estimates are biased toward the starting value of the adjustable response line. However, unlike the central tendency bias, this effect did not increase with load. This finding is significant for studies that utilize similar methodologies in which people must remember a stimulus and reproduce its value using a reproduction stimulus that has a value along the relevant stimulus dimension. For instance, consider a situation where the target line is slightly larger than the mean and the start line is greater than the target line. Also consider a target line that is slightly shorter than the mean and the start line is shorter than the target line. In these cases, the anchoring effects and the central tendency bias work in opposite directions. It is for this reason that we analyze the effects jointly in the same model, and the fact that they can both be detected speaks to their robustness. We hope that future studies employ an improved design: a random start length with a mean equal to the target line length, and have begun a more formal investigation on these effects (Duffy \& Smith, in preparation).

Note that, unlike Epley and Gilovich (2006), we did not find a relationship between CL and the anchoring effects in our experiments. Despite this, our experiments exhibited a variety of relationships between $\mathrm{CL}$ and adjustment (the absolute difference between the start and response line). In Experiment 2, we found that participants under high CL adjusted significantly less than they did under low CL. However, we found no such a relationship in Experiment 1. To the extent that a high CL can simulate the effect of having a lower cognitive ability, we look to the correlational literature. Bergman, Ellingsen, Johannesson, and Svensson (2010) found a relationship between measures of cognitive ability and anchoring; however, Oechssler, Roider, and Schmitz (2009) found no such a relationship. Perhaps a design better suited to studying anchoring effects would identify a relationship between anchoring and CL. Understanding the influence of anchoring effects in tasks where people respond by adjusting a stimulus to match a memory, such as in spatial location estimation tasks (i.e., Holden, Newcombe, \& Shipley, 2013; Huttenlocher et al., 1991) may help differentiate the relative influence of anchoring effects and the central tendency bias.

Our results suggest that further work is necessary to better understand the central tendency bias. First, it may be useful to consider individual differences in working memory. People vary in the size of their working memory capacity, which has significant consequences on performance in memory tasks (Frensch \& Miner, 1994; Unsworth \& Engle, 2007). It may be useful to study whether the effect of a CL manipulation is stronger in individuals with limited working memory capacity. Second, as mentioned, our design does not allow us to distinguish between the conjecture that $\mathrm{CL}$ affects either the encoding or the retrieval of a stimulus. A potential design that could distinguish between these two conjectures would manipulate the time during which the participant is under CL: during encoding or retrieval. We hope that future work can address this issue.

To summarize, we present the first evidence that cognitive load affects the central tendency bias in memory and that the central tendency bias is subject to anchoring effects. Together these results suggest that the effect of the stimulus distributions, which are presumably tied to specific stimulus dimensions, can be mediated by the high-level, general variable of cognitive load. These findings are important for those seeking to understand how cognitive constraints effect memories and interact with perception to guide behavior. Further research is needed to understand the mechanisms through which cognitive load influences behavior.

\section{References}

Akaike, H. (1974). A new look at the statistical model identification. IEEE Transaction on Automatic Control, 19, 716-723. doi:10. 1109/TAC.1974.1100705

Allen, R. J., Baddeley, A. D., \& Hitch, G. J. (2006). Is the binding of visual features in working memory resource-demanding? Journal of Experimental Psychology: General, 135, 298-313. 
Allred, S., Duffy, S., \& Smith, J. (2016). Cognitive load and strategic sophistication. Journal of Economic Behavior and Organization, $125,162-178$.

Ashourian, P., \& Loewenstein, Y. (2011). Bayesian inference underlies the contraction bias in delayed comparison tasks. PLoS ONE, 6 , e19551. doi:10.1371/journal.pone.0019551

Bergman, O., Ellingsen, T., Johannesson, M., \& Svensson, C. (2010). Anchoring and cognitive ability. Economics Letters, 107, 66-68. doi:10.1016/j.econlet.2009.12.028

Block, R. A., Hancock, P. A., \& Zakay, D. (2010). How cognitive load affects duration judgments: A meta-analytic review. Acta Psychologica, 134, 330-343.

Cocchi, L., Toepel, U., De Lucia, M., Martuzzi, R., Wood, S. J., Carter, O., \& Murray, M. M. (2011). Working memory load improves early stages of independent visual processing. Neuropsychologia, 49, 92-102.

Cornelissen, G., Dewitte, S., \& Warlop, L. (2011). Are social value orientations expressed automatically? Decision making in the dictator game. Personality \& Social Psychology Bulletin, 37, 1080-1090.

Crawford, L. E., Huttenlocher, J., \& Engebretson, P. H. (2000). Category effects on estimates of stimuli: Perception or reconstruction. Psychological Science, 11, 280-284. doi:10.1111/1467-9280.00256

Crawford, L. E., Huttenlocher, J., \& Hedges, L. V. (2006). Withincategory feature correlations and Bayesian adjustment strategies. Psychonomic Bulletin \& Review, 13, 245-250. doi:10.3758/ BF03193838

Duffy, S., \& Crawford, L. E. (2008). Primacy or recency effects in the formation of inductive categories. Memory \& Cognition, 36, 567577. doi:10.3758/MC.36.3.567

Duffy, S., Huttenlocher, J., Hedges, L. V., \& Crawford, L. E. (2010). Category effects on stimulus estimation: Shifting and skewed frequency distributions. Psychonomic Bulletin \& Review, 17, 224-230. doi:10.3758/PBR.17.2.224

Duffy, S., Huttenlocher, J., \& Crawford, L. E. (2006). Children use categories to maximize accuracy in estimation. Developmental Science, 9, 597-603. doi:10.1111/j.1467-7687.2006.00538.x

Duffy, S., \& Smith, J. (2014). Are there brains in games? Cognitive load in the multiple player prisoner's dilemma game. Journal of Behavioral \& Experimental Economics, 47-56. doi: 10.1016/j. socec.2014.01.006

Epley, N., \& Gilovich, T. (2006). The anchoring-and-adjustment heuristic: Why the adjustments are insufficient. Psychological Science, 17(4), 311-318.

Frensch, P. A., \& Miner, C. S. (1994). Effects of presentation rate and individual differences in short-term memory capacity on an indirect measure of serial learning. Memory \& Cognition, 22, 95-110.

Furnham, A., \& Boo, H. C. (2011). A literature review of the anchoring effect. Journal of Socio-Economics, 40(1), 35-42.

Gilbert, D. T., Pelham, B. W., \& Krull, D. S. (1988). On cognitive busyness: When person perceivers meet persons perceived. Journal of Personality \& Social Psychology, 54, 733-740.

Goldstone, R. (1994). Influences of categorization on perceptual discrimination. Journal of Experimental Psychology: General, 23, 178200.

Hemmer, P., \& Steyvers, M. (2009a). A Bayesian account of reconstructive memory. Topics in Cognitive Science, 1, 189-202.

Hemmer, P., \& Steyvers, M. (2009b). Integrating episodic memories and prior knowledge at multiple levels of abstraction. Psychonomic Bulletin \& Review, 16(1), 80-87.

Hinson, J. M., Jameson, T. L., \& Whitney, P. (2003). Impulsive decision making and working memory. Journal of Experimental Psychology: Learning, Memory, \& Cognition, 29, 298-306.

Holden, M. P., Newcombe, N. S., \& Shipley, T. F. (2013). Location memory in the real world: Category effects in 3 dimensional space. Cognition, 128, 45-55.
Huttenlocher, J., Hedges, L. V., \& Duncan, S. (1991). Categories and particulars: prototype effects in estimating spatial location. Psychological Review, 98, 352-376. doi:10.1037/0033-295X.98.3.352

Huttenlocher, J., Hedges, L. V., \& Vevea, J. L. (2000). Why do categories affect stimulus judgment? Journal of Experimental Psychology: General, 129, 220-241.

Jazayeri, M., \& Shadlen, M. N. (2010). Temporal context calibrates interval timing. Nature Neuroscience, 13, 1020-1026. doi:10.1038/ nn. 2590

LeBoeuf, R. A., \& Shafir, E. (2006). The long and short of it: Physical anchoring effects. Journal of Behavioral Decision Making, 19, 393 406.

Mann, T., \& Ward, A. (2007). Attention, self-control, and health behaviors. Current Directions in Psychological Science, 16, 280-283. doi: 10.1111/j.1467-8721.2007.00520.x

Martin, L. L., Seta, J. J., \& Crelia, R. A. (1990). Assimilation and contrast as a function of people's willingness and ability to expend effort in forming an impression. Journal of Personality and Social Psychology, 59, 27-37.

Morey, C. C., \& Bieler, M. (2013). Visual short-term memory always requires general attention. Psychonomic Bulletin \& Review, 20, $163-170$

Morey, C. C., \& Cowan, N. (2004). When visual and verbal memories compete: Evidence of cross-domain limits in working memory. Psychonomic Bulletin \& Review, 11, 296-301.

Oechssler, J., Roider, A., \& Schmitz, P. W. (2009). Cognitive abilities and behavioral biases. Journal of Economic Behavior \& Organization, 72, 147-152. doi:10.1016/j.jebo.2009.04.018

Olkkonen, M., \& Allred, S. R. (2014). Short-term memory affects color perception in context. PLOS ONE, 9(1), e86488. doi:10.1371/ journal.pone. 0086488

Olkkonen, M., McCarthy, P. F., \& Allred, S. R. (2014). The central tendency bias in color perception: Effects of internal and external noise. Journal of Vision, 14, 1-15.

Poulton, E. C. (1989). Bias in quantifying judgements. New York, NY: Taylor \& Francis.

Sailor, K. M., \& Antoine, M. (2005). Is memory for stimulus magnitude Bayesian? Memory \& Cognition, 33, 840-851.

Shiv, B., \& Fedorikhin, A. (1999). Heart and mind in conflict: The interplay of affect and cognition in consumer decision making. Journal of Consumer Research, 26, 278-292.

Swann, W. B., Hixon, G., Stein-Seroussi, A., \& Gilbert, D. T. (1990). The fleeting gleam of praise: Cognitive processes underlying behavioral reactions to self-relevant feedback. Journal of Personality \& Social Psychology, 59, 17-26.

Unsworth, N., \& Engle, R. W. (2007). The nature of individual differences in working memory capacity: Active maintenance in primary memory and controlled search from secondary memory. Psychological Review, 114, 104-132.

Van den Bos, K., Peters, S. L., Bobocel, D. R., \& Ybema, J. F. (2006). On preferences and doing the right thing: Satisfaction with advantageous inequity when cognitive processing is limited. Journal of Experimental Social Psychology, 42, 273-289.

Ward, A., \& Mann, T. (2000). Don't mind if I do: Disinhibited eating under cognitive load. Journal of Personality and Social Psychology, $78,753-763$.

Wigboldus, D. H., Sherman, J. W., Franzese, H. L., \& van Knippenberg, A. (2004). Capacity and comprehension: Spontaneous stereotyping under cognitive load. Social Cognition, 22, 292-309. doi:10.1177/ 0272989X09341751

$\mathrm{Xu}$, J., \& Griffiths, T. L. (2010). A rational analysis of the effects of memory biases on serial reproduction. Cognitive Psychology, 60, $107-126$.

Zokaei, N., Heider, M., \& Husain, M. (2014). Attention is required for maintenance of feature binding in visual working memory. The Quarterly Journal of Experimental Psychology, 67, 1191-1213. 